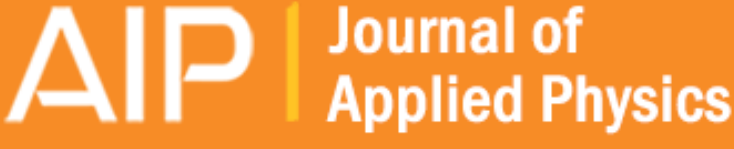

\section{Origin of high piezoelectric response in A-site disordered morphotropic phase boundary composition of lead-free piezoelectric $0.93(\mathrm{Na0} .5 \mathrm{Bi} 0.5) \mathrm{TiO}-0.07 \mathrm{BaTiO}$}

Deepam Maurya, M. Murayama, A. Pramanick, W. T. Reynolds Jr., Ke An, and Shashank Priya

Citation: Journal of Applied Physics 113, 114101 (2013); doi: 10.1063/1.4792729

View online: http://dx.doi.org/10.1063/1.4792729

View Table of Contents: http://scitation.aip.org/content/aip/journal/jap/113/11?ver=pdfcov

Published by the AIP Publishing

\section{Articles you may be interested in}

Polar nanoregions and dielectric properties in high-strain lead-free 0.93(Bi1/2Na1/2)TiO3-0.07BaTiO3

piezoelectric single crystals

J. Appl. Phys. 115, 014105 (2014); 10.1063/1.4861030

Direct evidence of correlations between relaxor behavior and polar nano-regions in relaxor ferroelectrics: A case

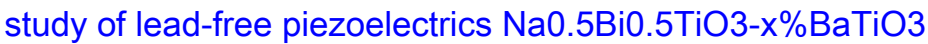

Appl. Phys. Lett. 103, 241914 (2013); 10.1063/1.4846655

Origin of large recoverable strain in $0.94(\mathrm{Bi} 0.5 \mathrm{Na} 0.5) \mathrm{TiO}-0.06 \mathrm{BaTiO} 3$ near the ferroelectric-relaxor transition Appl. Phys. Lett. 102, 062902 (2013); 10.1063/1.4790285

Enhanced piezoelectricity and nature of electric-field induced structural phase transformation in textured leadfree piezoelectric $\mathrm{Na} 0.5 \mathrm{Bi} 0.5 \mathrm{TiO} 3-\mathrm{BaTiO} 3$ ceramics Appl. Phys. Lett. 100, 172906 (2012); 10.1063/1.4709404

Origin of the large strain response in ( $\mathrm{K} 0.5 \mathrm{Na} 0.5$ ) NbO 3 -modified ( Bi $0.5 \mathrm{Na} 0.5$ ) TiO 3 - BaTiO 3 lead-free piezoceramics

J. Appl. Phys. 105, 094102 (2009); 10.1063/1.3121203

\section{MIT LINCOLN}

LABORATORY CAREERS

Discover the satisfaction of innovation and service

to the nation
- Space Control

- Air \& Missile Defense

- Communications Systems \& Cyber Security

- Intelligence, Surveillance and

Reconnaissance Systems

- Advanced
Electronics
- Tactical Systems
- Homeland
Protection
- Air Traffic Control

LINCOLN LABORATORY

Massachusetts Institute of TeChNOLOgY

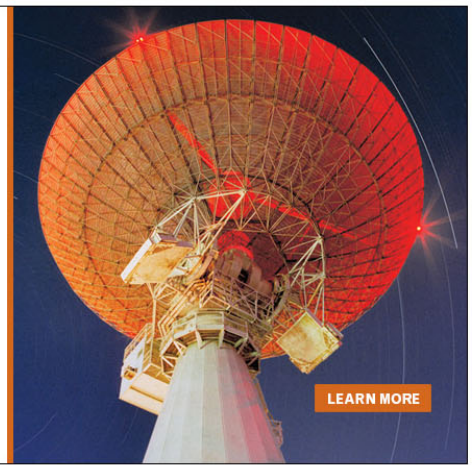




\title{
Origin of high piezoelectric response in A-site disordered morphotropic phase boundary composition of lead-free piezoelectric 0.93 $\left(\mathrm{Na}_{0.5} \mathrm{Bi}_{0.5}\right) \mathrm{TiO}_{3}-0.07 \mathrm{BaTiO}_{3}$
}

\author{
Deepam Maurya, ${ }^{1, a)}$ M. Murayama, ${ }^{2,3}$ A. Pramanick, ${ }^{4}$ W. T. Reynolds, Jr., ${ }^{2}$ Ke An, ${ }^{4}$ \\ and Shashank Priya ${ }^{1, a)}$ \\ ${ }^{1}$ Center for Energy Harvesting Materials and Systems (CEHMS), Bio-Inspired Materials and Devices \\ Laboratory (BMDL),Virginia Tech, Blacksburg, Virginia 24061, USA \\ ${ }^{2}$ Department of Materials Science and Engineering, Virginia Tech, Blacksburg, Virginia 24061, USA \\ ${ }^{3}$ Institute for Critical Technology and Applied Sciences, Virginia Tech, Blacksburg, Virginia 24061, USA \\ ${ }^{4}$ Chemical and Engineering Materials Division, Oak Ridge National Laboratory, Oak Ridge, Tennessee \\ 37831-2008, USA
}

(Received 24 December 2012; accepted 4 February 2013; published online 15 March 2013)

\begin{abstract}
Perovskite piezoelectric compositions near the morphotropic phase boundary (MPB) are known to exhibit high piezoelectric response. In lead-based $\mathrm{ABO}_{3}$ compound with $\mathrm{B}$-site disorder, the origin of this enhancement has been associated with the presence of an intermediate monoclinic/ orthorhombic state that bridges the adjacent ferroelectric rhombohedral and tetragonal phases. However, the origin of high piezoelectric response in lead-free $\mathrm{ABO}_{3}$ compounds with A-site disorder has not been conclusively established. We describe a microscopic model derived from comparative analyses of high resolution transmission electron microscopy and neutron diffraction that explains the origin of high piezoelectric response in lead-free MPB compositions of $0.93\left(\mathrm{Na}_{0.5} \mathrm{Bi}_{0.5}\right) \mathrm{TiO}_{3}-0.07 \mathrm{BaTiO}_{3}$. Direct observation of nanotwins with monoclinic symmetry confirmed the presence of an intermediate bridging phase that facilitates a pathway for polarization reorientation. Monoclinic distortions of an average rhombohedral phase are attributed to localized displacements of atoms along the non-polar directions. (C) 2013 American Institute of Physics. [http://dx.doi.org/10.1063/1.4792729]
\end{abstract}

\section{INTRODUCTION}

Environmental restrictions in many parts of the world are demanding the elimination of lead from all consumer electronics. This has prompted global investigations to find lead-free piezoelectric compositions with high electromechanical coupling. In the past decade, enhanced piezoelectric properties have been discovered in $\left(\mathrm{Na}_{0.5} \mathrm{Bi}_{0.5}\right) \mathrm{TiO}_{3}-\left(\mathrm{K}_{0.5} \mathrm{Bi}_{0.5}\right) \mathrm{TiO}_{3}$ (NBT-KBT) and $\left(\mathrm{Na}_{0.5} \mathrm{Bi}_{0.5}\right) \mathrm{TiO}_{3}-\mathrm{BaTiO}_{3}$ (NBT-x\%BT) compositions close to morphotrophic phase boundary (MPB). Particularly, NBT with R3c symmetry has emerged as a rhombohedral substitute for the lead-based counterpart similar to that of $\mathrm{PbZrO}_{3}$ in $\mathrm{Pb}(\mathrm{Zr}, \mathrm{Ti}) \mathrm{O}_{3}{ }^{1,2}$ However, some recent studies have indicated that the NBT, the end member in NBT-BT phase diagram, instead exhibits a monoclinic Cc symmetry. ${ }^{3,4}$ The solid solution $(1-\mathrm{x}) \mathrm{Na}_{0.5} \mathrm{Bi}_{0.5} \mathrm{TiO}_{3}-\mathrm{xBaTiO}_{3}$ (NBT-BT) exhibits MPB for $\mathrm{x}=0.05-0.07$. The magnitude of electromechanical coefficients measured at MPB for NBT-BT are $d_{33}=125 \mathrm{pC} / \mathrm{N}, d_{31}=40 \mathrm{pC} / \mathrm{N}, k_{31}=0.19$, and $\varepsilon_{11}^{T} / \varepsilon_{o}=733 .{ }^{1}$ However, the origin of piezoelectric property enhancement in lead-free $\mathrm{ABO}_{3}$ compounds near MPB with A-site compositional disorder is still being investigated.

Earlier theoretical and diffraction studies on lead-based solid solutions established the relation between the presence of an intermediate phase with monoclinic/orthorhombic symmetry near the MPB and the enhancement in piezoelectric

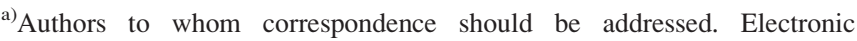
addresses: mauryad@vt.edu and spriya@vt.edu.
}

properties. The intermediate phase bridges the end-members of a phase diagram with tetragonal and rhombohedral symmetries. Bellaiche et al..$^{5}$ have proposed that for a monoclinic symmetry, the polarization can easily adjust along the applied electric field providing strong coupling between the degrees of freedom for polarization and crystallographic strain that leads to high piezoelectric response in $\mathrm{Pb}(\mathrm{Zr}, \mathrm{Ti}) \mathrm{O}_{3}$. Subsequent X-ray diffraction (XRD) studies confirmed the importance of a monoclinic phase during polarization rotation. ${ }^{6}$ For example, when electric field is applied along the pseudocubic [001] direction of rhombohedral PZN-PT with polarization vector along pseudocubic [111], the zero field rhombohedral state irreversibly changes first to a monoclinic $\mathrm{M}_{\mathrm{C}}$ phase and then to an orthorhombic phase with $\mathrm{a}_{\mathrm{m}}=\mathrm{c}_{\mathrm{m}}$. ${ }^{7}$ This intermediate monoclinic phase facilitates continuous rotation of polarization vector between the $T\langle 001\rangle$ and $R$ $\langle 111\rangle$ directions resulting in enhanced electromechanical response under external stimuli. Traditionally, at the MPB, rhombohedral and tetragonal phases are assumed to coexist as the space group symmetries of these two ferroelectric phases are not connected by any direct group theory correlation. ${ }^{8,9}$ The enhanced piezoelectric response in these compositions was attributed to the co-existence of different ferroelectric phases at the MPB. Additionally, such type of two-phase coexistence has been considered to give rise to residual strains at the heterophase boundaries, which could lead to lowering of symmetry at smaller length scales. ${ }^{10}$

Unlike lead-based ferroelectrics that have varying occupancies on the B-site of an $\mathrm{ABO}_{3}$ compound, NBT-based 
piezoelectrics exhibit disorder on the A-site. Different sitespecific disorders can lead to lowering of crystal symmetry in $\mathrm{ABO}_{3}$ compounds at nano-scales. For example, in wellknown $\mathrm{Pb}(\mathrm{Zr}, \mathrm{Ti}) \mathrm{O}_{3}$ with $\mathrm{B}$-site disorder, a monoclinic phase was evident from synchrotron $\mathrm{X}$-ray diffraction under applied electric field. Establishment of a long-range monoclinic order in $\mathrm{Pb}(\mathrm{Zr}, \mathrm{Ti}) \mathrm{O}_{3}$ could be explained as a condensation of local displacements of $\mathrm{Pb}$ ions along non polar directions in the tetragonal phase. ${ }^{11}$ In $\mathrm{ABO}_{3}$ perovskite type systems, the A-site cation has stronger interaction with oxygen anions and hence a change in the A-site cation can perturb the crystal symmetry. The different interionic interactions can be understood by considering the differences in the local environment of $\mathrm{A}$ and $\mathrm{B}$ cations in the perovskite structure. In the case of $\mathrm{Pb}(\mathrm{Zr}, \mathrm{Ti}) \mathrm{O}_{3}$, for A-site cations, the oxygen nearest neighbor shell has 12 -fold symmetry. This symmetry is however broken for the B-cation next nearest neighbor shell. $\mathrm{Zr}$ is a larger ion than $\mathrm{Ti}$, and thereby purely repulsive interaction between $\mathrm{Pb}$ and $\mathrm{B}$ cation $\left(\mathrm{Zr}^{4+} / \mathrm{Ti}^{4+}\right)$ has been considered to be stronger for $\mathrm{Pb}-\mathrm{Zr}$ than $\mathrm{Pb}-\mathrm{Ti}{ }^{12}$ On the other hand, all B-cation distribution does not display a strong dependence on the local structure and are aligned closely with the $\mathrm{Pb}$ ion distributions. ${ }^{12}$ Grinberg et al. ${ }^{12}$ considered $\mathrm{Pb}$ distortions, produced by a hierarchy of interactions, as the determining factor for the average structure of the material. The effect of disorder on the different sites in the perovskite structure is schematically illustrated in Figure 1.

Figure 1(a) shows the schematic of long range ordering due to cooperative displacement of $\mathrm{Ti}$ in archetype piezoelectric $\mathrm{BaTiO}_{3}$. The displacement of $\mathrm{B}$-site cation is shown by arrows. However, when $\mathrm{B}$-site is substituted by $\mathrm{Zr}^{4+}$ and $\mathrm{Ti}^{4+}$, the local displacement of $\mathrm{Pb}^{2+}$ is substantially perturbed by the presence of the neighboring ions. The $\mathrm{Pb}^{2+}$ tends to go away from $\mathrm{Zr}^{4+}$ and close to the $\mathrm{Ti}^{4+}$. However, on average, $\mathrm{Pb}^{2+}$ distortions were found to be in $[001]_{\mathrm{c}}$ direction ${ }^{12}$ leading to long range monoclinic phase in $\mathrm{Pb}(\mathrm{Zr}, \mathrm{Ti}) \mathrm{O}_{3}$ at MPB. The schematic representations of ionic displacements in $\mathrm{Pb}(\mathrm{Zr}, \mathrm{Ti}) \mathrm{O}_{3}$ [Ref. 12] are depicted in Figure 1(b). The perforated circles and small arrows are showing the tendency of $\mathrm{Pb}^{2+}$ displacements and $\mathrm{Ti} / \mathrm{Zr}$ displacements in polar direction, respectively.
The situation in NBT-BT is more complex because the A-site is occupied by three types of ions $\left(\mathrm{Bi}^{3+}, \mathrm{Ba}^{2+}\right.$, and $\mathrm{Na}^{+}$) with different chemistry like charge, ionic radius, and electronegativity, etc., as illustrated in Figure 1(c). The B-site $\mathrm{Ti}^{4+}$ would have stronger repulsive interaction if surrounded predominantly by the $\mathrm{Bi}^{3+}$. This interaction would vary significantly depending on the type of neighboring ions $\mathrm{Ba}^{2+}$ or $\mathrm{Na}^{+}$. As discussed above, the A-site cation has stronger interaction with oxygen anions and hence a change in the A-site cation can strongly perturb the crystal symmetry. These local structural distortions could reduce local symmetry.

For NBT-BT systems of MPB compositions, local-scale monoclinic symmetry from an overall rhombohedral symmetry has been proposed from diffused anelastic and dielectric spectra, nuclear magnetic resonance (NMR) spectra and diffuse X-ray diffraction patterns. ${ }^{13,14}$ However, no direct microstructural evidence for such localized distortions has been reported. Therefore, it is still debatable whether the perceived significance of local monoclinic distortions, as observed in lead-based piezoelectrics, could be extended as a general principle to other systems as well. Here, we provide direct evidence from high-resolution transmission electron microscope (HR-TEM) and neutron diffraction measurements for localized monoclinic distortion of an overall rhombohedral symmetry in NBT-BT in the form of nanoscale twins. The importance of the nanotwins towards enhanced piezoelectric properties is discussed.

An understanding of the crystallographic distortions near the MPB also could help to clarify the phase transition behaviors that have been reported for NBT-BT. During cooling from high temperature, NBT undergoes a sequence of phase transitions from a high temperature cubic $(\mathrm{C})$ phase to ferroelastic tetragonal (T) phase at $540^{\circ} \mathrm{C}$, and a diffuse phase transition (DPT) from ferroelastic tetragonal (T) to rhombohedral (R) phase between $200{ }^{\circ} \mathrm{C}$ and $320^{\circ} \mathrm{C} .{ }^{15}$ Different variants of phase diagrams for NBT-BT have been proposed that consist of ferroelectric rhombohedral and tetragonal phases as end components, and an antiferroelectric (AFE) or ferrielectric orthorhombic component as an intermediate phase.,16-19 Coexistence of rhombohedral and tetragonal phases has been observed over a broad range of temperatures and $E$-fields while the orthorhombic phase was proposed to exhibit AFE-
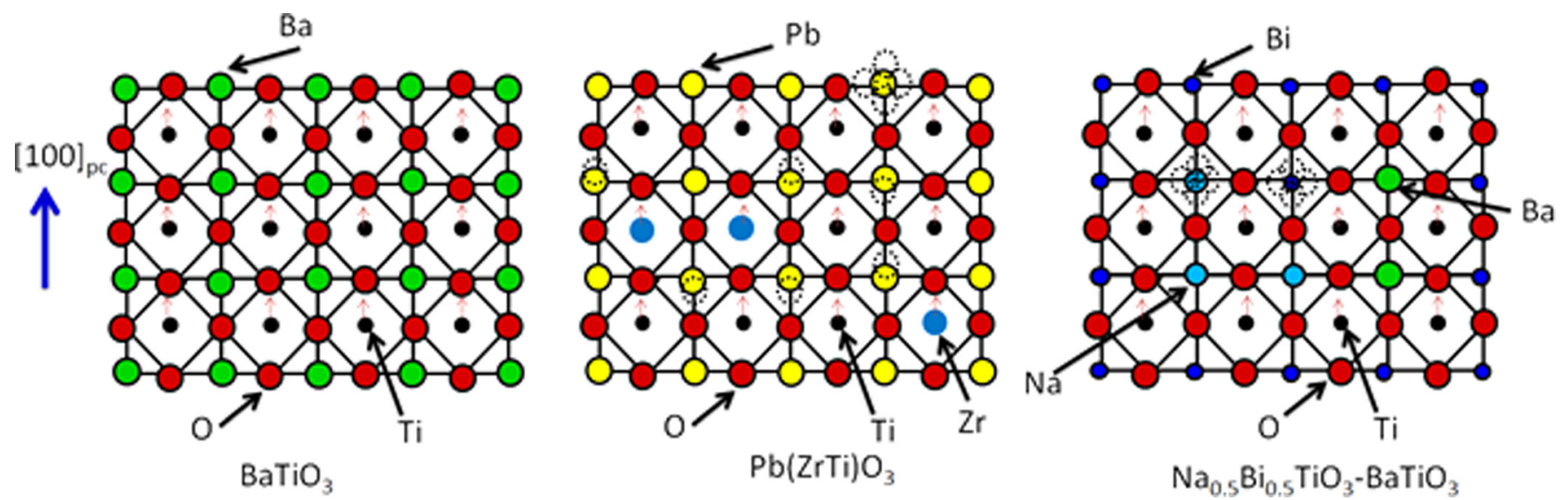

FIG. 1. (a) Long range polar lattice distortions in $\mathrm{BaTiO}_{3}$, (b) polar lattice distortions in $\mathrm{Pb}(\mathrm{ZrTi}) \mathrm{O}_{3}$, (c) polar lattice distortions in $\mathrm{Na}_{0.5} \mathrm{Bi}_{0.5} \mathrm{TiO}_{3}-\mathrm{BaTiO}_{3}$. The dotted circles marked represent possible distortion direction of A-site atoms depending on the neighboring atoms. 
like characteristics. ${ }^{20,21}$ Recent studies on the orthorhombic phase describe it as a relaxor phase with polar nanoregions. $^{22,23}$ The high piezoelectric response and $E$-field induced strain in NBT based systems has been attributed to structural phase transitions under the application of an E-field. ${ }^{24}$ However, in a previous study, we have demonstrated that $E$-field induced structural phase transition is not prerequisite for achieving high piezoelectric constant in NBT$\mathrm{BT} .{ }^{25}$ Intriguingly, textured and randomly oriented polycrystalline ceramics of the same composition of NBT-BT were found to exhibit drastically different phase transition behaviors under electric field and were tentatively associated with variance in coherence lengths of polar nanoregions and internal stresses induced by domain switching.

Based on these earlier results, it could be said that the role of nanoregions in the NBT-based system is quite special as compared to lead-based relaxors. For example, in the case of PZN-0.1PT, the off-centering of $\mathrm{Pb}$ cations containing a lone pair of electrons plays an important role in the origin of polar nano regions and has been found to favor the polar displacement of corresponding nearest B-site cations. ${ }^{26}$ During the application of $E$-field, the correlated displacement of $\mathrm{Pb}$ ions could be considered to result in long range polar phase with lower symmetry. However, as discussed earlier, the case is very different in NBT-BT system with the A-site being shared by $\mathrm{Bi}, \mathrm{Na}$, and $\mathrm{Ba}$. The nature of local polar distortions created by $\mathrm{Bi}$ on the A-site containing a lone pair of electron would be different than that due to $\mathrm{Na}$ and $\mathrm{Ba}$ without lone pair of electrons in terms of cation off-centering and octahedral tilting disorder. Therefore, in NBT-BT, the local distortions due to the substitution on A-site are more complex than that of B-site substituted lead-based relaxors and would behave differently under external stimuli. These short range local structural distortions can further couple with elastic degrees of freedom resulting in hierarchical domain structures from mesoscopic lamellar domains to polar nano regions. ${ }^{27,28}$ Moreover, NBT-BT exhibits a high temperature unique ferroelastic phase transition followed by low temperature ferroelectric phase transition as discussed earlier. The ferroelastic domain variants created during the high temperature ferroelastic phase transitions sustains up to room temperature and coexists with the nano sized ferroelectric domains. ${ }^{29}$ The nano sized ferroelectric domains were found to be geometrically and elastically restricted within micro-sized ferroelastic domains. ${ }^{29}$ Therefore, the percolation behavior of these polar nano regions under the influence of $E$-field in piezoelectric Asite disordered NBT-BT system can be influenced by the presence of ferroelastic domains. However, this kind of transition has not been reported in B-site disordered lead-based relaxor like $\mathrm{Pb}(\mathrm{Zr}, \mathrm{Ti}) \mathrm{O}_{3}$ (PZT). Also, the percolation behavior of polar nano regions vary significantly with the different systems, as PZN-0.8PT was not found to show significant change in polar nano regions (PNRs) under applied $E$-field. ${ }^{30}$ We think that the percolation behavior and coupling between local polar distortions with the nonlinear elastic lattice ${ }^{31}$ play an important role in NBT based systems exhibiting interesting functional response and structural phase transition behavior.

In this work, we have carried out structural investigations on high purity single crystals and polycrystalline samples. We have chosen different types of samples for our studies in order to account for the different types of perturbation of domain morphology expected in them. For polycrystalline samples, whisker morphology was developed to clearly delineate the contribution from the crystallography. Most of the piezoelectric materials used in polycrystalline form consist of a large number of randomly oriented grains each with multiple domains of varying orientations as allowed by crystallographic symmetry. ${ }^{32}$ The domain switching in one grain is constrained by differently oriented neighboring grains. Moreover, neighboring grains can also induce lateral stress on each other during the paraelectric to ferroelectric (PE-FE) phase transition. ${ }^{33}$ Therefore, the perturbation of domain behavior due to interaction with the differently oriented neighboring grains is a highly correlated collective process that is complicated to quantify. ${ }^{32}$ In order to overcome this problem, NBT-BT whiskers were synthesized with a diameter of $\sim 2-3$ grains (grain size ranging $300-500 \mathrm{~nm}$ ) as shown in the inset of Fig. 4.With this morphology, the influence on the domain system in a given grain from the domains in neighboring grain will be negligible.

\section{EXPERIMENTAL}

Single crystals were grown using the molten flux method. $^{34}$ In order to grow single crystals of 0.93 $\left(\mathrm{Na}_{0.5} \mathrm{Bi}_{0.5}\right) \mathrm{TiO}_{3}-0.07 \mathrm{BaTiO}_{3}$ (after this point NBT-BT will refer to this MPB composition), pre-calcined (800$900{ }^{\circ} \mathrm{C}(2 \mathrm{~h})$ ) powder was mixed with $30 \mathrm{wt}$. $\% \mathrm{Bi}_{2} \mathrm{O}_{3}$ and 10 wt. $\% \mathrm{Na}_{2} \mathrm{CO}_{3}$ and heated in sealed Pt crucible at $1300{ }^{\circ} \mathrm{C}$ for $6 \mathrm{~h}$ before slowly cooling down to room temperature (RT). The synthesis of $\mathrm{Na}_{2} \mathrm{Ti}_{6} \mathrm{O}_{13}$ (NTO) whiskers was performed by using the molten salt synthesis method as reported earlier. $^{35}$ These NTO whiskers were used to synthesize NBT-BT whiskers of the same composition. ${ }^{36}$ The MPB composition $\left[0.93\left(\mathrm{Na}_{0.5} \mathrm{Bi}_{0.5}\right) \mathrm{TiO}_{3}-0.07 \mathrm{BaTiO}_{3}\right]$ of single crystals and whiskers was confirmed by energy dispersive spectroscopy. The XRD experiments were conducted on these NBT-BT whiskers (thickness $\sim 0.5-1.0 \mu \mathrm{m}$ and length $\sim 10-20 \mu \mathrm{m}$ ) to confirm the formation of perovskite phase at RT. These whiskers were pressed into pellet and sintered at $1200^{\circ} \mathrm{C}$ for $2 \mathrm{~h}$. The electromechanical coupling constants for various variants of NBT-BT like single crystals, whisker based polycrystalline ceramics, and traditionally processed NBT-BT ceramics are listed in Table I. The specimen processed using NBT-BT whiskers shows higher piezoelectric response than traditionally processed variant. For morphological investigations, Zeiss LEO 1550 scanning electron microscope was used. The selected area electron diffraction (SAED) patterns were recorded using Philips

TABLE I. List of the piezoelectric properties for various variants of leadfree piezoelectric NBT-0.07BT.

\begin{tabular}{lcc}
\hline \hline Sample & $d_{33}(\mathrm{pC} / \mathrm{N})$ & $k_{31}(\%)$ \\
\hline NBT-0.07BT (single crystal) & $300 \pm 5$ & $40 \pm 2$ \\
NBT-0.07BT (whisker-based) & $205 \pm 5$ & $28 \pm 2$ \\
NBT-0.07BT (conventional sintering) & $160 \pm 5$ & $18 \pm 2$ \\
\hline \hline
\end{tabular}



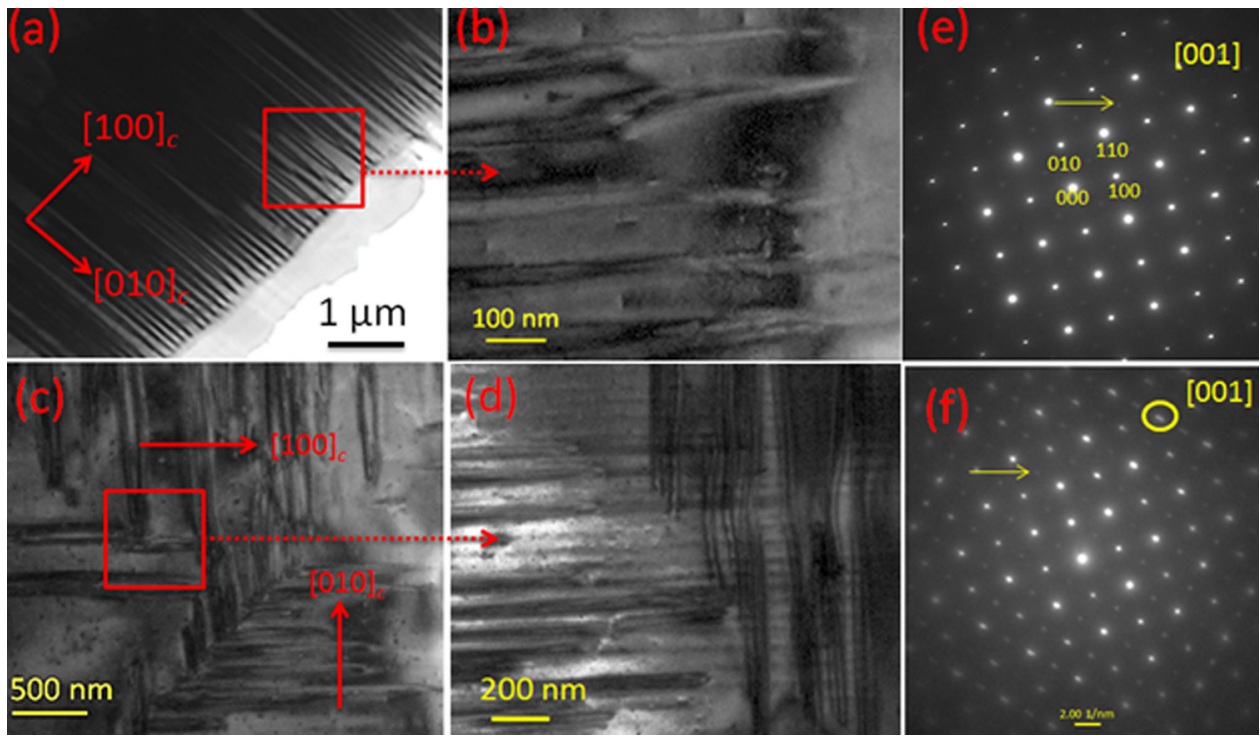

FIG. 2. High purity single crystal studies. (a) TEM image of thin domains with domain walls planar on (100), the magnified view of these domains is depicted in (b). (c) The herringbone type domains structure composed of thin domains with planar domain walls on (100) and (010) and intersecting on (110) plane. The magnified view of these domains is depicted in (d). (e) SAED with large probe size and (f) electron diffraction with nano beam. The arrows marked in (e) and (f) indicate superlattice reflections. It was noticed that $\boldsymbol{g}_{100} / \boldsymbol{g}_{010}=1$ and the angle between these vectors was $\sim 90^{\circ}$, however, the SAED with nano beams shows deviation in the ratio and the angles indicating lowering of symmetry.

EM420 transmission electron microscope. HR-TEM and nano-beam diffraction analysis was performed by using FEI Titan 80-300 transmission electron microscope. The highresolution neutron powder diffraction patterns of the NBTBT whiskers were collected at the VULCAN diffractometer of the Spallation Neutron Source (SNS), Oak Ridge National Laboratory. ${ }^{37}$ The sample was loaded into a cylindrical vanadium can and the diffraction patterns were collected with an incident neutron beam in the time-of-flight mode. Rietveld refinement on the diffraction pattern was performed using the GSAS software package. ${ }^{38}$

\section{RESULTS AND DISCUSSION}

We first look at the twin microstructure in the single crystals and whiskers of NBT-BT. During transition from a higher symmetry phase to a lower symmetry phase, twin boundaries play an important role as twin boundaries are considered to be localized pseudo symmetries relating one twin domain to another by a lost symmetry operation. The twin domains are formed by the loss of point group symmetry, and the interaction between twin boundaries (walls) can give rise to a multitude of domain patterns creating difference between the local atomic structure of twin (domain) boundary and bulk. ${ }^{39}$ The arrangement of domain patterns are hierarchical because it can be decomposed into simpler patterns until the most elementary unit "twin wall" or "domain wall," is found. ${ }^{39}$

The multitude of domain structures in NBT-BT single crystal is depicted in the bright field TEM images of Figures 2(a)-2(d) and 3. The ferroelastic lamellar domains having widths $\sim 100-200 \mathrm{~nm}$ with planar $\{100\}_{C}$ domain walls can be observed in Figure 2(a). The magnified view of these lamellar domains is depicted in Fig. 2(b). A HR-TEM image of lattice fringes across domains and fast Fourier transform (FFT) patterns of two regions in the image are presented in Figure S1(b) [figure provided in supplementary file ${ }^{40}$ ]. The FFT and nano probe electron diffraction patterns ${ }^{40}$ (Fig. S1(d)) recorded on the two sides of different contrast regions of these domains were found to be similar indicating no significant structural changes across them. The bigger lamellar domains with planar domain walls on $(100)_{C}$ and $(010)_{C}$ intersect at angle $\sim 90^{\circ}$ with plane of intersection $\{\overline{1} 10\}_{C}$ giving rise to a herringbone-type domain structure as observed in modulated systems ${ }^{41}$ (marked with the rectangle in Fig. 2(c)). The magnified image of these domains indicates that the thicker lamellar domains are composed of smaller domains (Fig. 2(d)). The lamellar domain consists of two domains with a common domain wall attracting each other and this force of attraction decreases rapidly with the increase in the distance between the two domain walls ${ }^{42}$ resulting in fine substructure ranging from submicron to nano scale in dimension that is represented as hierarchical structure of ferroelastic domains. Similar hierarchical domain structures were reported for ferroelastic lead phosphate. ${ }^{43}$ Another region of interest showing domain structure is depicted in Figure 3, which shows polydomain plates with domain boundaries on different planes. In this image along with the larger size lamellar domains with thickness $\sim 100$ $200 \mathrm{~nm}$ having domain walls planar on $\{100\} \mathrm{c}$, we also observed another set of domain variants with smaller size domains $(\sim 50 \mathrm{~nm})$ with boundaries planar on $\{1 \overline{1} 0\}_{c}$. The thickness of polydomain plate $\delta$ having domains thickness $d$

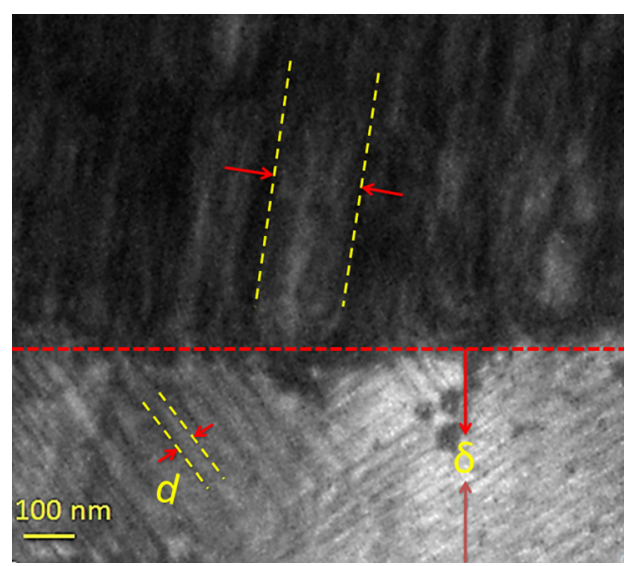

FIG. 3. Bright field TEM image of various domain variants of NBT-BT single crystals viewed from $[001]_{\mathrm{c}}$ zone axis. 
is marked in Fig. 3, which is $\sim 400 \mathrm{~nm}$. Hierarchical patterns with lamellar domains are formed depending on the interaction between domains and the energy of the domain walls. According to Khachaturyan et al., ${ }^{44}$ the typical domain size in ferroelastic materials is related to the thickness of the poly domain plate $\delta$ and domain wall energy density $\gamma_{t}$ as:

$$
d=\xi \sqrt{\frac{\gamma_{t}}{\mu \varepsilon_{o}^{2}}} \delta
$$

where $\xi$ is a dimensionless constant, $\mu$ is the shear modulus, and $\varepsilon_{o}$ is the twinning strain. This theory was extended to piezoelectric materials to explain miniaturized domain state and adaptive phase in MPB compositions ${ }^{45}$ and the thickness $d$ of ferroelectric domains in a polydomain plate of thickness " $\delta$ " was found to be proportional to $\sqrt{\gamma_{t} \delta}$. On decreasing the domain wall energy, the wall thickness would decrease. ${ }^{44}$ Furthermore, Theissmann et al. ${ }^{46}$ investigated change in domain structure across MPB of PZT. They reported miniaturized domain in MPB composition of PZT with polydomain plate (microdomain) having thickness $\delta \sim 250 \mathrm{~nm}$ containing nano-sized domains. ${ }^{46}$ These nanodomains were found to respond to $E$-field instead of microdomains.

The crystallographic details for the nanodomains are next elucidated from the electron diffraction patterns. Figure 2(e) shows a SAED pattern from the NBT-BT single crystal with $[001]_{C}$ zone axis with electron beam probe size $\sim 400 \mathrm{~nm}$. The $1 / 2\{\text { ooe }\}_{C}$ ( $e$ stands for even Miller indices and $o$ for odd Miller indices) super-lattice reflections are marked with arrow in Fig. 2(e). In this case, the ratio of the magnitude of $\boldsymbol{g}$ vectors $\left(g_{100} / g_{010}=1\right)$ was found to be unity with $90^{\circ}$ between them. Interestingly, from the diffraction patterns recorded using nano beam $(\sim 5 \mathrm{~nm})$ probe (Fig. $2(\mathrm{f})$ ), we observe deviations from the unity ratio of $\boldsymbol{g}$ vectors, as well as the angle between the $\boldsymbol{g}$ vectors deviates from $90^{\circ}$. This suggests a lowering of symmetry locally $\left[\boldsymbol{g}_{100} / \boldsymbol{g}_{010}=0.98\right.$ and angle between them deviates by $0.75^{\circ}-1.0^{\circ}$ (please note these deviations are much clearer when observed over several diffraction spots)]. The implications of this local deviation in crystal symmetry will be more apparent in later discussions. Also, the splitting in the higher order diffraction spots reveals the presence of twins with small shear displacement $\sim 0.03$ calculated from the splitting of higher order diffraction spot in Fig. 2(f). Similar magnitude of splitting in electron diffraction patterns was observed for PZT. ${ }^{47}$ Such small shear displacements could be the result of only partial relaxation of stress in NBT-BT single crystals as there are various crystallographically allowed domain variants with collective interactions among them. It is therefore important to use NBT whiskers with diameter of only few grains so that interactions due to surrounding domain variants are reduced.

Next, HR-TEM analysis was performed on NBT-BT whiskers (inset of Figure 4). HR-TEM analysis was repeated across several different samples and sample area to confirm the results. Figure 4 shows the HR-TEM image of lattice fringes exhibiting the presence of nanotwins. Yellow lines are drawn along several lattice planes as a visual guide to highlight the presence of a twin boundary. For comparison, FFT patterns were calculated from a twin free region (region
1) and the twinned region (region 2). These FFT patterns could not be indexed using a rhombohedral symmetry as has been suggested in earlier works (such as Refs. 1 and 2). Interestingly, both the FFT patterns can be indexed using a monoclinic symmetry (JCPDS \#460001). The lattice parameters of this monoclinic cell are: $a=0.5553(2), b=0.6675$ (3), $\mathrm{c}=0.5520(1) \mathrm{nm}, \beta=120.54(2)^{\circ}$. The monoclinic crystal structure within the nanotwins is significant since monoclinic lattice can have more degrees of freedom to adapt local stress field in ceramics. ${ }^{47}$

The zone axis $[2 \overline{2} 1]_{M}$ (M stands for monoclinic phase) was obtained from the indexed FFT pattern in Figure 4. The FFT pattern of twinned region clearly depicts an additional diffraction spot near $(\overline{1} 02)_{M}$ and the inter-planer angle was measured to be $11^{\circ}$. The twinning plane (or "mirror plane") was parallel to the zone axis and is the boundary between two twinned domains. Next, we calculated the twin plane/ twin boundary, which was found to be $(\overline{1} \overline{1} 0)_{\mathrm{M}}$. The continuity of lattice planes across the boundary indicates that the twins were coherent. The arrow marked with $\mathrm{N}$ in the FFT pattern of region 2 points towards the shapes of twin spots. Two spots very close to each other suggest the presence of nanotwins with very small twinning shear of $\sim 0.06$. These nanotwins were present within boundaries of bigger nanotwin shown by yellow lines. Similar nanotwin structure with thickness of about one atomic layer was observed in nanocrystalline copper. ${ }^{48}$ The smaller size of nanotwins has been found to be associated very small twin boundary energy ${ }^{44}$ as discussed earlier. According to Khachaturyan et al., ${ }^{44}$ the lower surface energy and large strain energy lead to smaller size nano twins. However, at certain smaller sizes (comparable to atomic scale size), the finite interaction among the twin walls constrains further miniaturization of twin domains. Randall et al.$^{49}$ calculated the surface energy $(\sigma)$ of $\{100\}_{\mathrm{R}}$ and $\{110\}_{\mathrm{R}}$ twins in rhombohedral system following the work of Zirnov ${ }^{50}$ and suggested that $\sigma_{100} \approx 3 \sigma_{110}$. Therefore, the formation $\{110\}$ twins is more energetically favorable than that of $\{100\}$ twins.

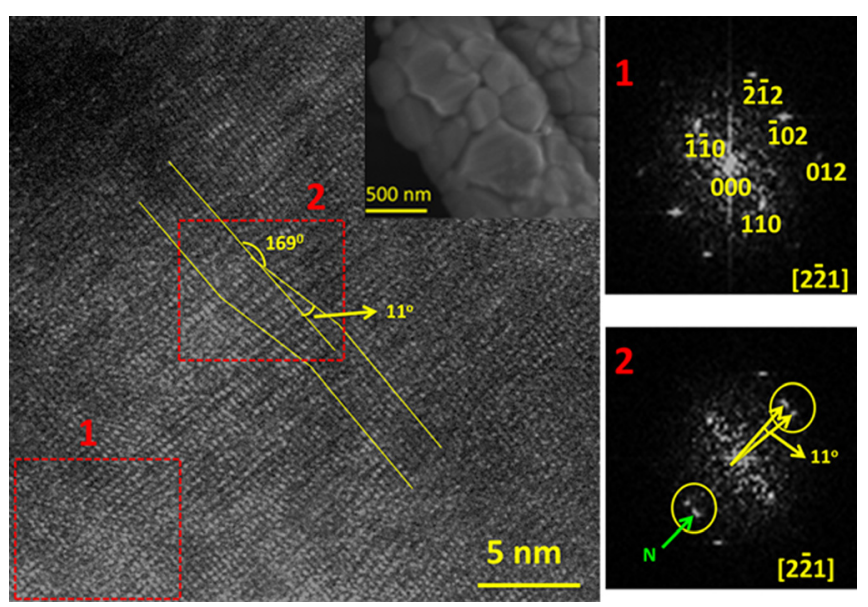

FIG. 4. A HR-TEM image showing the presence of a nanometer twinned region in NBT-BT whiskers. The two patterns on the right labeled 1 and 2 are FFT patterns from the corresponding regions of the HR-TEM image marked 1 and 2; the split spots in the yellow circles of the FFT of region 2 indicates twinning of the atomic planes. The inset on the HR-TEM image is a SEM micrograph of NBT-BT whisker. 

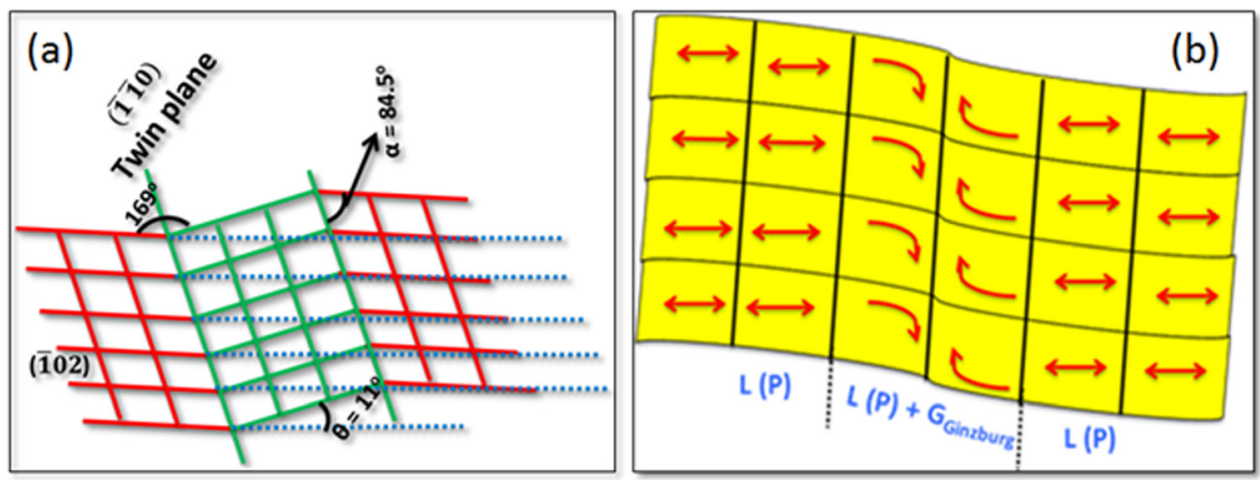

FIG. 5. (a) A schematic representation of the twin arrangement observed in Fig. 4, (b) schematic plot of bent lattice planes indicating the various energy contributions [After Salje ${ }^{43}$ ]. The lattice on either end of the plot is distorted due to PE-FE phase transition. The spontaneous strain is symbolized by the double arrows. The relevant energy is Landau potential. When the planes are bent additional gradient energy occurs as described by the Ginzburg energy. The single arrow represents bending of the planes.

The schematic representation of twinning is depicted in Figure 5(a). In the present case, twinning gives rise to plane with same interplanar spacing but slightly deviated $\left(11^{\circ}\right)$ from the parent plane. The magnitude of shear can be given by $\tan \theta=\tan \left(11^{\circ}\right)=0.19$, which is much higher than that of twinning shear (0.06) of nanotwins having thickness of atomic layer as discussed above. The magnitude of shear ( $~ 0.19)$ observed in this case was comparable to martensitic materials. ${ }^{51}$ The orientation of habit plane with respect to the parent lattice plane at a given point can be estimated geometrically ${ }^{52}$ as: $\sin \alpha=\frac{\sin \theta}{\sqrt{2-2 \cos \theta}}=84.5^{\circ}$ for $\theta=11^{\circ}$. Furthermore, the crystallographic symmetry of NBT-BT whiskers at larger length scales was characterized from neutron diffraction. The high resolution neutron diffraction pattern was fitted separately for two possible space groups$R 3 c$ and $C c$. The rhombohedral space group $R 3 c$, with antiphase oxygen octahedral tilts, has been reported previously for NBT-BT solid solutions. ${ }^{15,18}$ The space group $C c$ is a monoclinic subgroup of the $R 3 c$ which also allows tilting of the oxygen octahedral, and has been mentioned in a previous report. ${ }^{3}$ The Rietveld refinement for space group $R 3 c$ yielded the following lattice parameters: $a_{H}=0.54999$ (2) $\mathrm{nm}$, $\mathrm{c}_{\mathrm{H}}=1.35583(9) \mathrm{nm}, \mathrm{V}=0.35519$ (2) $\mathrm{nm}^{3}$, with $\mathrm{Rp}=0.057$, $\mathrm{wRp}=0.0738$ and reduced $\chi^{2}=3.415$; the starting variables for the fitting were taken from Jones and Thomas ${ }^{15}$ (Figure 6(a)). In comparison, Rietveld refinement for space group $C c$ yielded the following lattice parameters: $\mathrm{a}_{\mathrm{M}}=0.95368$ (11), $\mathrm{b}_{\mathrm{M}}=0.54921$ (7), $\mathrm{c}_{\mathrm{M}}=0.55154$ (7), $\beta=124.956$ (8), with $\mathrm{Rp}=0.072, w \mathrm{Rp}=0.097$, and reduced $\chi^{2}=5.909$ (Figure 6(b)); the initial setting for this refinement was taken from $C c$ structure of PZT. ${ }^{53}$ The $R 3 c$ space group yielded better fit to the diffraction pattern which is evident from a $20 \%$ improvement in the value of $\mathrm{Rp}$ for the $R 3 c$ phase. Furthermore, examination of the high intensity (111) peak (see insets) clearly reveals the better fit for the R3c model. A mixture of tetragonal and rhombohedral phases was also tested; however, it did not provide a stable fit.

Neutron diffraction of NBT-BT whiskers indicates an average rhombohedral structure with $R 3 C$ space group in contrast to monoclinic symmetry observed in HR-TEM analysis. Therefore, although the material has a monoclinic symmetry at a local scale, it retains an average rhombohedral structure, as revealed by bulk powder neutron diffraction. It was observed that even though the $R 3 c$ model provides a good fit to the neutron diffraction profile in terms of overall statistics, slight broadening of the measured Bragg peaks with respect to the calculated pattern was observed (for example see peaks 111 and 3/2 1/2 1/2 in Figure 6). Such broadening could arise from local monoclinic distortions of the crystal in the matrix. This is consistent with the previous report in which polar nano regions of $\sim 20 \mathrm{~nm}$ were estimated from diffuse $\mathrm{X}$-ray scattering, although the average structure retained $R 3 C$ symmetry. ${ }^{54}$ It was suggested from the diffused $\mathrm{X}$-ray scattering measurements that the displacements of $\mathrm{Bi}^{3+}, \mathrm{Ba}^{2+}$, and $\mathrm{Na}^{+}$away from $[111]_{\mathrm{R}}$ ( $\mathrm{R}$ stands for rhombohedral phase) direction towards $[100]_{\mathrm{T}}$ (T stands for tetragonal phase), can give rise to local monoclinic symmetry.

A lowering of the crystal symmetry within the twin domains at the nanoscale suggests that they are formed due to the loss of point group symmetry during the phase transition from the high temperature paraelastic cubic phase to an intermediate ferroelastic tetragonal phase in NBT based systems. Thus, recently observed structural phase transition in NBT based materials ${ }^{55}$ under the application of electric field could be expected to be facilitated by the presence of nano twins. Next, we recorded SAED from a different zone axis to investigate the nature of superlattice reflections in NBT-BT whiskers. Figure S2 depicts the SAED patterns ${ }^{40}$ taken along various zone axes on NBT-BT whiskers. The SAED patterns with zone axes $[001]_{C}$ and $[112]_{C}$ depicts superlattice reflections $1 / 2\{\text { ooe }\}_{C}$ and $1 / 2\{\text { ooo }\}_{C}(e$ stands for even miller indices and $o$ for odd miller indices) as marked in Figs. S2(a) and S2(b), respectively. ${ }^{40}$ The observance of $1 / 2\{\text { ooe }\}_{C}$ and $1 / 2\{o o o\}_{C}$ superlattice reflections are consistent with the average rhombohedral phase with the $R 3 c$ space group. ${ }^{56,57}$ However, the origin of super lattice reflections can be attributed to disorder in octahedral tilting and cation ordering, ${ }^{58}$ both of which could lead to local departure from the average rhombohedral symmetry ${ }^{59}$ resulting in the formation of a localized monoclinic phase $\left(\mathrm{Schematic}^{40}\right.$ showing cation displacement and antiphase octahedra tilting is depicted in Fig. S3) with lower symmetry as observed in HR-TEM analysis. However, these local distortions with various tilting disorder and ionic displacements are compensated over longer length scales resulting in an average anti-phase tilting of octahedra in the rhombohedral structure. ${ }^{58,60}$ The local displacements of the A-site cations along $[100]_{\mathrm{T}}$ are also compensated over larger length scales giving rise to an average rhombohedral symmetry. This is illustrated through a simple model showing representation of $[001]_{C}$ projection of the perovskite structure is provided in Figure 7(a). The octahedra tilting are 

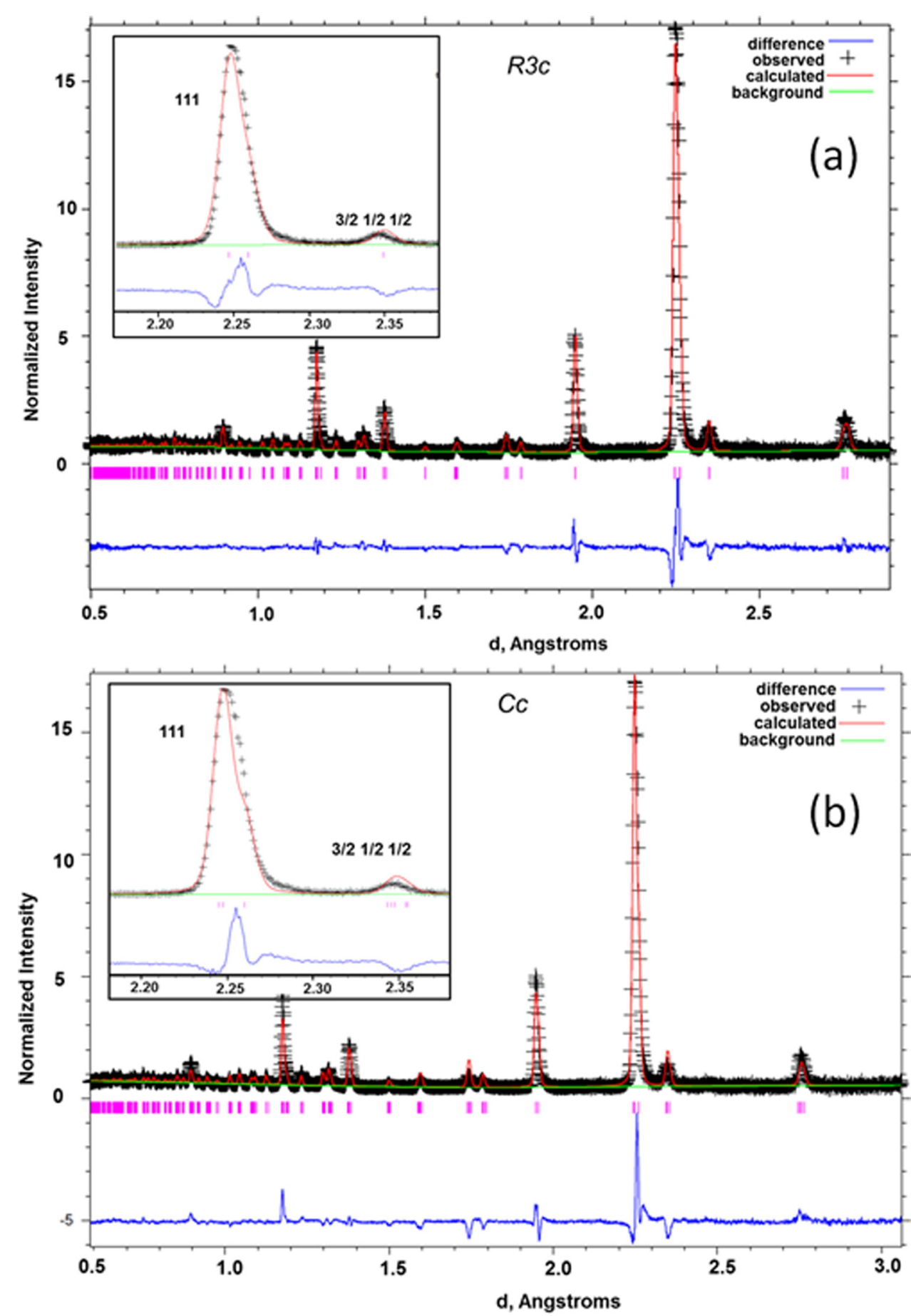

FIG. 6. High resolution neutron diffraction patterns fitted with space group (a) $\mathrm{R} 3 \mathrm{c}$ and (b) Cc. Insets are showing $\{111\}$ reflection was fitted well R3c space group. not shown in the schematic model. The unit cell is marked by the square with broken lines. The simulated SAED patterns based on rhombohedral phase with the $R 3 c$ space group equivalent to $[001]_{C}$ zone axis is presented in Fig. 7(b). It can be noticed that the displacement of A-site cations along $[100]_{C}$ would lead to distortion in the lattice consequently affecting the diffraction pattern leading to lowering of symmetry locally. However, as discussed above, these distortions averaged over larger length scales lead to higher average symmetry as observed in high resolution neutron diffraction. Moreover, the small size of nanotwins gives rise to high density of twin boundaries, which makes material more deformable as twin boundaries can accommodate the macroscopic strain, induced by external electric field or applied stress. ${ }^{61}$ The rearrangement of nanotwins with symmetry lower than the macroscopic symmetry results in giant strain and enhanced piezoelectric response under applied fields. ${ }^{62}$ Furthermore, we believe that higher mobility of these nano twins is responsible for giving rise to high strain energy density in MPB composition of NBT-BT. ${ }^{63,64}$ Recently, in-situ TEM experiments under electrical biasing have also shown that in $\mathrm{PZN}$, polarization reversal can occur by reorientation of nanoscale non- $180^{\circ}$ domain walls between monoclinic nanotwins. ${ }^{65}$

The local crystallographic distortions in the MPB composition of NBT-BT can be justified from thermodynamic 


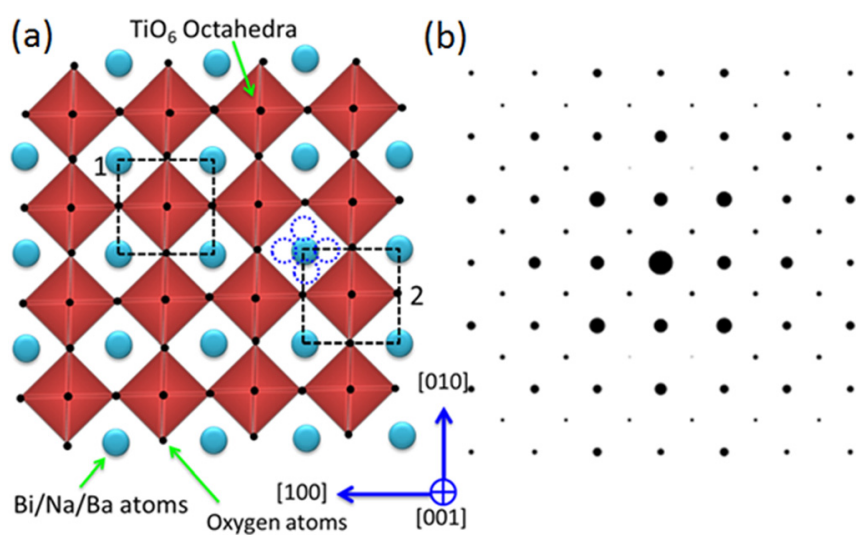

FIG. 7. (a) A simple model showing $[001]_{\mathrm{pc}}$ projection of $\mathrm{ABO}_{3}$ type structure. The unit cell is marked as square 1 with broken lines. The displacement of A-site (indicated by solid circle with light blue color) cation depicted by the dotted circle in the unit cell 2, which could lead to lower local symmetry of the system. (b) The simulated SADP using R3c unit cell with [001 $]_{\mathrm{pc}}$ zone axis.

considerations as explained below. NBT-BT is a ferroelasticferroelectric material, therefore, thermodynamic description of NBT-BT requires at least two order parameters; polarization $P_{1}$ and strain $P_{2}$. The different symmetry for high temperature ferroelastic (tetragonal) and low temperature ferroelectric (rhombohedral) phase requires biquadratic coupling between the order parameters as reported by Salje. ${ }^{43}$ The variation of the order parameters $P(\mathrm{r})$ in space requires that the Ginzburg energy $\left(F_{G}\right)$ term should be added to Landau potentials $\left(F_{L}\right)$ and the Landau-Ginzburg potential for NBT-BT could be written as

$$
F=F_{o}+F_{G}+F_{L},
$$

where $F_{G}=\frac{a}{2}\left(\nabla P_{1}\right)^{2}+\frac{b}{2}\left(\nabla P_{2}\right)^{2}$ and

$$
\begin{aligned}
F_{L}= & \frac{1}{2} \alpha_{1} P_{1}^{2}+\frac{1}{4} \beta_{1} P_{1}^{4}+\frac{1}{6} \gamma_{1} P_{1}^{6}+\frac{1}{2} \alpha_{2} P_{2}^{2} \\
& +\frac{1}{4} \beta_{2} P_{2}^{4}+\frac{1}{6} \gamma_{2} P_{2}^{6}+\lambda P_{1}^{2} P_{2}^{2}
\end{aligned}
$$

$F_{o}$ is the energy in unconstrained paraelectric state. If the sign of coupling parameter $(\lambda)$ is negative, the two order parameters strengthen each other and lower the Gibbs free energy as reported by Salje. ${ }^{43}$ In this case, the temperature is considered to be a control parameter. However, positive coupling parameter depicts competition between two order parameters and increases free energy. ${ }^{43}$ The nature of coupling between these order parameters could modulate the order of the phase transition resulting in diffused phase transitions similar to that of NBT-BT systems. Figure 5(b) represents a schematic depicting lattice distortion. As shown in Fig. 5(b), the Landau potential causes the atoms to be displaced in the low-symmetry phase and Ginzburg energy leads to spatial variation of solitary waves. $^{43}$ In the case of negative Ginzburg energy, the total Gibbs free energy is reduced by each twin boundary and the system tends to nucleate more twin boundaries to increase thermodynamic stability. However, the density of twin boundaries is controlled by the repulsion potential experienced by them resulting in incommensurately modulated phase at nano scale in terms of local monoclinic distortions observed in NBT-BT.

\section{SUMMARY AND CONCLUSIONS}

The local structural distortions coupled with elastic degrees of freedom results in hierarchical domain structures in MPB composition NBT-BT. The local polar distortions with lower symmetry in A-site disordered NBT-BT systems are different from those of lead based B-site disordered relaxors and so as their percolation under external stimuli. The dynamics of ferroelectric nano domains could also be considerably constrained by ferroelastic domains in NBT-BT with unique high temperature ferroelastic phase transition. The nano beam electron diffraction revealed higher deviation in $g$ vectors suggesting lower local symmetry, which was however, found to be compensated over larger length scales. To delineate the contribution from interdomain interactions and surrounding numerous grains, we investigated polycrystalline NBT-BT whiskers with same compositions. The HRTEM analysis revealed presence of a local monoclinic symmetry as monoclinic lattice with higher degree of freedom can easily adopt local stress. However, the neutron diffraction analysis suggested an average rhombohedral phase. Interestingly, we directly observed monoclinic nanotwins (nanodomains) in NBT-BT whiskers. The monoclinic domains, inside rhombohedral domains, can easily transform to tetragonal domains and thereby act as the bridging phase facilitating the polarization rotation. Moreover, the small size of nanotwins could make the material more deformable due to the easy accommodation of the macroscopic strain induced by external stimuli. The nanotwins with higher mobility will result in high strain density and large electromechanical response in the MPB compositions of NBT-BT. These results emphasize the generic role of nanotwins with reduced symmetry towards enhanced piezoelectric properties of MPB compositions. The short range crystallographic distortions in NBT-BT can explain different phase transitions that have been reported for this material around the MPB.

\section{ACKNOWLEDGMENTS}

The authors gratefully acknowledge the financial support from National Science Foundation (Nano whisker Synthesis and Property measurements) and Office of Basic Energy Sciences, U.S. Department of Energy \#DE-FG0207ER46480 (Microscopy analysis). The authors would also like to thank the Nanoscale Characterization and Fabrication Laboratory, ICTAS, VT for their help in characterization (HR-TEM). Neutron diffraction measurements at Oak Ridge National Laboratory's Spallation Neutron Source were sponsored by the Scientific User Facilities Division, Office of Basic Energy Sciences, U.S. Department of Energy.

${ }^{1}$ T. Takenaka, K.-i. Maruyama, and K. Sakata, Jpn. J. Appl. Phys., Part 1 30, 2236 (1991).

${ }^{2}$ J. Rodel, W. Jo, K. T. P. Seifert, E. M. Anton, and T. Granzow, J. Am.

Ceram. Soc. 92, 1153 (2009).

${ }^{3}$ S. Gorfman and P. Thomas, J. Appl. Crystallogr. 43, 1409 (2010). 
${ }^{4}$ E. Aksel, J. S. Forrester, J. L. Jones, P. A. Thomas, K. Page, and M. R. Suchomel, Appl. Phys. Lett. 98, 152901 (2011).

${ }^{5}$ L. Bellaiche, A. García, and D. Vanderbilt, Phys. Rev. Lett. 84, 5427 (2000).

${ }^{6}$ B. Noheda, D. E. Cox, G. Shirane, R. Guo, B. Jones, and L. E. Cross, Phys. Rev. B 63, 014103 (2000).

${ }^{7}$ B. Noheda, D. E. Cox, G. Shirane, S.-E. Park, L. E. Cross, and Z. Zhong, Phys. Rev. Lett. 86, 3891 (2001).

${ }^{8}$ W. Cao and L. E. Cross, Phys. Rev. B 47, 4825 (1993).

${ }^{9}$ M. J. Hoffmann, M. Hammer, A. Endriss, and D. C. Lupascu, Acta Mater. 49, 1301 (2001).

${ }^{10}$ V. Yu. Topolov, J. Appl. Phys. 111, 094109 (2012).

${ }^{11}$ R. Guo, L. E. Cross, S.-E. Park, B. Noheda, D. E. Cox, and G. Shirane, Phys. Rev. Lett. 84, 5423 (2000).

${ }^{12}$ I. Grinberg, V. R. Cooper, and A. M. Rappe, Nature 419, 909 (2002).

${ }^{13}$ F. Cordero, F. Craciun, F. Trequattrini, E. Mercadelli, and C. Glassi, Phys. Rev. B 81, 144124 (2010).

${ }^{14}$ I. P. Aleksandrova, A. A. Sukhovsky, Yu. N. Ivanov, Yu. E. Yablonskaya, and S. B. Vakhrushev, Phys. Solid State 50, 496 (2008).

${ }^{15}$ G. O. Jones and P. A. Thomas, Acta Crystallogr., Sect. B: Struct. Sci. 58, 168 (2002).

${ }^{16}$ B. Wylie-Van Eerd, D. Damjanovic, N. Klein, N. Setter, and J. Trodahl, Phys. Rev. B 82, 104112 (2010).

${ }^{17}$ J. Yao, L. Yan, W. Ge, L. Luo, J. Li, and D. Viehland, Phys. Rev. B 83, 054107 (2011)

${ }^{18}$ W. Jo, J. E. Daniels, J. L. Jones, X. Tan, P. A. Thomas et al., J. Appl. Phys. 109, 014110 (2011).

${ }^{19}$ J. E. Daniels, W. Jo, J. Rödel, V. Honkimaki, and J. L. Jones, Acta Mater. 58, 2103 (2010)

${ }^{20}$ V. Dorcet, G. Trolliard, and P. Boullay, Chem. Mater. 20, 5061 (2008).

${ }^{21}$ G. Trolliard and V. Dorcet, Chem. Mater. 20, 5074 (2008).

${ }^{22}$ W. Jo, S. Schaab, E. Sapper, L. A. Schmitt, H.-J. Kleebe et al., J. Appl. Phys. 110, 074106 (2011).

${ }^{23}$ C. Ma, X. Tan, E. Dul'kin, and M. Roth, J. Appl. Phys. 108, 104105 (2010).

${ }^{24}$ Y. Guo, Y. Liu, R. L. Withers, F. Brink, and H. Chen, Chem. Mater. 23, 219 (2011).

${ }^{25}$ D. Maurya, A. Pramanick, K. An, and S. Priya, Appl. Phys. Lett. 100, 172906 (2012).

${ }^{26}$ N. Waeselmann, B. Mihailova, B. J. Maier, C. Paulmann, M. Gospodinov, V. Marinova, and U. Bismayer, Phys. Rev. B 83, 214104 (2011).

${ }^{27}$ K. H. Ahn, T. Lookman, and A. R. Bishop, Nature 428, 401 (2004).

${ }^{28}$ G. Xu, J. Wen, C. Stock, and P. M. Gehring, Nature Mater. 7, 562 (2008).

${ }^{29}$ J. Yao, W. Ge, L. Luo, J. Li, D. Viehland, and H. Luo, Appl. Phys. Lett. 96, 222905 (2010).

${ }^{30}$ G. Xu, Z. Zhong, Y. Bing, Z.-G.Ye, and G. Shirane, Nature Mater. 5, 134 (2006).

${ }^{31}$ A. R. Bishop, T. Lookman, A. Saxena, and S. R. Shenoy, Europhys. Lett. 63, 289 (2003).

${ }^{32}$ J. Y. Li, R. C. Rogan, E. Ustundag, and K. Bhattacharya, Nature Mater. 4, 776 (2005).

${ }^{33}$ F. X. Li and R. K. N. D. Rajapakse, Acta Mater. 55, 6472-6480 (2007).

${ }^{34}$ Y. Hosono, K. Harada, and Y. Yamashita, Jpn. J. Appl. Phys., Part 140 , 5722 (2001).
${ }^{35}$ D. Maurya, M. Murayama, and S. Priya, J. Am. Cearm. Soc. 94, 2857 (2011).

${ }^{36}$ D. Maurya, V. Petkov, A. Kumar, and S. Priya, Daltan Trans. 41, 5643 (2012).

${ }^{37}$ X. L. Wang, T. M. Holden, G. Q. Rennich, A. D. Stoica, P. K. Liaw, H. Choo, and C. R. Hubbard, Phys. B: Condens. Matter 385-386, 673 (2006).

${ }^{38}$ A. C. Larson and R. B. Von Dreele, General Structure Analysis System (GSAS) (Los Alamos National Laboratory, NM, USA, 1995).

${ }^{39}$ E. K. H. Salje, S. A. Hayward, and W. T. Lee, Acta Crystallogr., Sect. A 61, 3 (2005).

${ }^{40}$ See supplementary material at http://dx.doi.org/10.1063/1.4792729 for HR-TEM, electron diffraction data, and Schematic of octahedral tilting.

${ }^{41}$ K. Parlinski, Comput. Phys. Rep. 8, 153 (1988).

${ }^{42}$ E. Salje, B. Kuscholke, and B. Wruck, Phys. Chem. Miner. 12, 132 (1985).

${ }^{43}$ E. K. H. Salje, Phase Transitions in Ferroelastic and Co-Elastic Crystlas (Cambridge University Press, Cambridge, 1990).

${ }^{44}$ A. G. Khachaturyan, S. M. Shapiro, and S. Semenovskaya, Phys. Rev. B 43, 10832 (1991).

${ }^{45}$ Y. M. Jin, Y. U. Wang, A. G. Khachaturyan, J. F. Li, and D. Viehland, Phys. Rev. Lett. 91, 197601 (2003).

${ }^{46}$ R. Theissmann, L. A. Schmitt, J. Kling, R. Schierholz, K. A. Schönau, and H. Fuess, J. Appl. Phys. 102, 024111 (2007).

${ }^{47}$ R. Schierholz and H. Fuess, J. Appl. Crystallogr. 45, 766 (2012).

${ }^{48}$ X. Z. Liao, Y. H. Zhao, S. G. Srinivasan, Y. T. Zhu, R. Z. Valiev, and D. V. Gunderov, Appl. Phys. Lett. 84, 592 (2004).

${ }^{49}$ C. A. Randall, D. J. Barber, and R. W. Whatmore, J. Mater. Sci. 22, 925 (1987).

${ }^{50}$ V. A. Zhirnov, Sov. Phys. JETP 35, 822 (1959)

${ }^{51}$ S. Ii, K. Yamauchi, Y. Maruhashi, and M. Nishida, Scr. Mater. 49, 723 (2003).

${ }^{52}$ J. F. Nie, Acta Mater. 52, 795 (2004).

${ }^{53}$ D. Pandey, A. K. Singh, and S. Baik, Acta Crystallogr., Sect. A 64, 192 (2008).

${ }^{54}$ J. Kreisel, P. Bouvier, B. Dkhil, P. A. Thomas, A. M. Glazer, T. R. Welberry, B. Chaabane, and M. Mezouar, Phys. Rev. B 68, 014113 (2003).

${ }^{55}$ M. Hinterstein, M. Knapp, M. Hölzel, W. Jo, A. Cervellino, H. Ehrenberg, and H. Fuess, J. Appl. Crystallogr. 43, 1314 (2010).

${ }^{56}$ A. M. Glazer, Acta Crystallogr., Sect. A 31, 756 (1975).

${ }^{57}$ D. I. Woodward and I. M. Reaney, Acta Crystallogr., Sect. B 61, 387 (2005).

${ }^{58}$ I. Levin and I. M. Reaney, Adv. Funct. Mater. 22, 3445 (2012).

${ }^{59}$ V. Dorcet and G. Trolliard, Acta Mater. 56, 1753 (2008).

${ }^{60}$ D. I. Woodward, J. Knudsen, and I. M. Reaney, Phys. Rev. B 72, 104110 (2005).

${ }^{61}$ Yu. U. Wang, Phys. Rev. B 73, 014113 (2006).

${ }^{62}$ S. Kaufmann, U. K. Rößler, O. Heczko, M. Wuttig, J. Buschbeck, L. Schultz, and S. Fähler, Phys. Rev. Lett. 104, 145702 (2010).

${ }^{63}$ C.-S. Tu, C.-M. Hsieh, R. R. Chien, V. H. Schmidt, F.-T. Wang, and W. S. Chnag, J. Appl. Phys. 103, 074117 (2008).

${ }^{64}$ W. S. Chang, L. C. Lim, P. Yang, H. Miao, C.-S. Tu, Q. Chen, and A. K. Soh, Appl. Phys. Lett. 94, 202907 (2009).

${ }^{65}$ Y. Sato, T. Hirayama, and Y. Ikuhara, Phys. Rev. Lett. 107, 187601 (2011). 